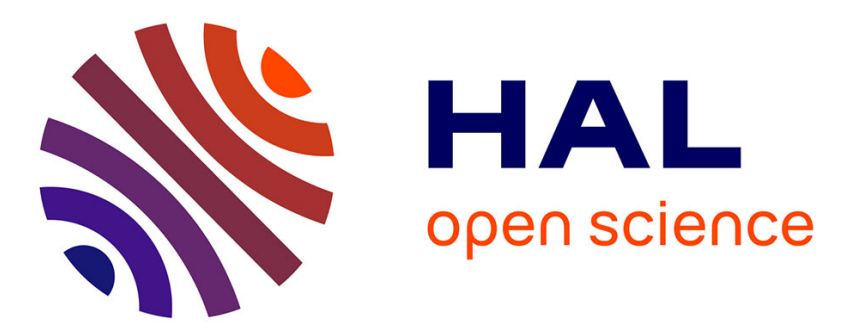

\title{
Travail social et demande d'asile: les enseignements d'une étude sur l'accueil des demandeurs d'asile dans les Alpes-Maritimes
}

Gilles Frigoli, Jessica Jannot

\section{- To cite this version:}

Gilles Frigoli, Jessica Jannot. Travail social et demande d'asile: les enseignements d'une étude sur l'accueil des demandeurs d'asile dans les Alpes-Maritimes. Revue française des affaires sociales, 2004, 4, pp.223-242. halshs-00079011

\section{HAL Id: halshs-00079011 https://shs.hal.science/halshs-00079011}

Submitted on 25 Oct 2006

HAL is a multi-disciplinary open access archive for the deposit and dissemination of scientific research documents, whether they are published or not. The documents may come from teaching and research institutions in France or abroad, or from public or private research centers.
L'archive ouverte pluridisciplinaire HAL, est destinée au dépôt et à la diffusion de documents scientifiques de niveau recherche, publiés ou non, émanant des établissements d'enseignement et de recherche français ou étrangers, des laboratoires publics ou privés. 


\title{
Travail social et demande d'asile : les enseignements d'une étude sur l'accueil des demandeurs d'asile dans les Alpes-Maritimes
}

\author{
Gilles Frigoli et Jessica Jannot*
}

\section{- Introduction}

Au cours des dernières années en France, l'hébergement des demandeurs d'asile est devenu l'objet de préoccupations grandissantes, tant pour les acteurs ou observateurs engagés aux côtés de ces populations ${ }^{1}$, que pour les administrations centrales en charge de la lutte contre les exclusions ${ }^{2}$. Les données du problème sont connues : une augmentation du nombre de demandes d'asile dans la période récente associée à un taux d'obtention du statut de réfugié très faible; des délais d'instruction des dossiers de demande d'asile importants ; un statut légal qui interdit de travailler; des aides financières prévues par la législation d'un montant insuffisant pour se tourner vers le parc social et, a fortiori, vers le marché locatif privé.

Des réponses ont été apportées. On sait que la capacité du dispositif national d'accueil (DNA) a été récemment augmentée afin de faire face à cette situation. Mais l'offre n'est pas à la hauteur des besoins et, comme le constate l'Observatoire national de la pauvreté et de l'exclusion sociale (Espinasse, 2002), le DNA est aujourd'hui saturé ( $c f$. encadré 1). Cette pression se reporte mécaniquement sur les lieux d'hébergement généralistes (centres d'hébergement et de réinsertion sociale (CHRS), accueils de nuit, hôtels meublés) qui, dans de nombreux centres urbains, permettent de répondre à la demande qui vient s'ajouter à celle des usagers «traditionnels » de ce type de structures et qui n'a pas diminué dans le même temps.

\footnotetext{
* Gilles Frigoli : maître de conférences en sociologie à l'université de Nice-Sophia-Antipolis et chercheur au sein du SOLIIS-URMIS (UMR CNRS 7032). Jessica Jannot : doctorante au sein du SOLIIS-URMIS.

1 Voir par exemple le rapport publié en 2003 à ce sujet par France Terre d'Asile (FTDA, 2003).

2 Le rapport récent de l'inspection générale des Affaires sociales (IGAS) consacré à l'évaluation de la loi du 29 juillet 1998 relative à la lutte contre les exclusions (IGAS, 2004) revient à plusieurs reprises sur cette question. Ainsi par exemple : « Dans le domaine de l'urgence, la mission constate la saturation persistante de l'offre d'hébergement d'urgence qui, malgré les efforts financiers engagés, est embolisée par l'augmentation continue du nombre de demandeurs d'asile » (op. cit., p. 3).
} 


\section{Encadré 1 : La situation du dispositif national d'accueil}

\section{- Le contexte}

En 2003, 9,8\% des 52204 premières demandes d'asile effectuées (hors mineurs accompagnants) ont donné lieu à une réponse positive de l'OFPRA. $\grave{A}$ titre indicatif, ce taux atteignait presque $80 \%$ en 1981 . Le nombre de primo-demandes est en augmentation constante depuis 1997 (21 416 primo-demandes enregistrées cette année-là). Source : OFPRA (2004).

Le délai moyen de traitement des dossiers était, en 2002, de 324 jours (Source: OFPRA, 2003). II a été ramené à quatre mois en 2003 (Source : OFPRA, 2004).

Depuis 1991, en France, les demandeurs d'asile ne sont plus autorisés à occuper un emploi. Les prestations financières accessibles aux demandeurs d'asile conventionnel ${ }^{1}$ sont l'allocation d'attente (un versement de 304 euros par adulte et 106 euros par enfant) et l'allocation d'insertion (280 euros par mois pendant une durée maximale de douze mois). Aucune aide n'est prévue pour les demandeurs d'asile territorial ${ }^{2}$.

\section{- Le dispositif}

Le dispositif national d'accueil (DNA) se décompose en centres d'accueil pour demandeurs d'asile (CADA) et centres provisoires d'hébergement $(\mathrm{CPH})$ destinés aux réfugiés. Le nombre de places en CADA a connu une augmentation sensible récemment puisqu'il s'élevait à 5282 en 2001 pour atteindre, à la fin 2002, le chiffre de 10317 places auxquelles s'ajoutent 1028 places en $\mathrm{CPH}$ (Source: IGAS, 2004). Toutefois, ces chiffres sont à rapporter aux 51000 demandes d'asile enregistrées et aux 8500 certificats de réfugié délivrés la même année par l'OFPRA. (Source: OFPRA, 2003).

Mais les difficultés que rencontrent les demandeurs d'asile ne concernent pas que le logement, il y aussi la nourriture, les vêtements, les soins, etc. Dès lors, il n'est guère étonnant que, dans les lieux où ils résident, les demandeurs d'asile et leur famille soient, pour nombre d'entre eux, amenés à fréquenter assidûment les divers lieux d'accueil, services sociaux et caritatifs, susceptibles de leur apporter de l'aide dans tous les domaines.

C'est ce que nous avons pu observer dans le département où nous avons enquêté. L'étude que nous avons menée était consacrée aux conditions d'hébergement des demandeurs d'asile, des réfugiés et des personnes déboutées du droit d'asile dans les Alpes-Maritimes ( $c f$. encadré 2). Mais nous avons rapidement constaté que, au-delà de l'offre d'hébergement,

1 Sur la réforme du droit d'asile du 10 décembre 2003 voir dans le même numéro la présentation de H. Furnon-Petrescu (N.D.L.R.).

2 Idem. 
c'est l'offre d'action sociale au sens large qui se trouvait sommée de faire face à l'émergence de nouveaux besoins auxquels les acteurs locaux n'étaient pas toujours préparés. À Nice, comme nous le verrons, c'est de manière soudaine et sous l'emprise de l'urgence que le «problème » des demandeurs d'asile fit son apparition dans le champ des thématiques qui, comme celles de l'enfance en danger, de l'accès aux soins ou du surendettement, alimentent la réflexion, et souvent les craintes, des acteurs locaux de l'action sociale. Suite à ce que beaucoup décrivent localement comme un " afflux massif » autant qu'imprévu de demandeurs d'asile, nombreuses sont les institutions et structures engagées dans la gestion des situations de précarité sociale qui durent trouver des solutions, élaborer une politique organisationnelle, parfois revoir leur positionnement au sein de l'architecture de l'offre d'action sociale. Mais la question de l'adaptation à cette nouvelle donne, celle des pratiques à mettre en œuvre face à ces nouveaux publics, s'imposa dans le même temps dans d'autres lieux que ceux où se décident les grandes orientations de la politique locale en matière d'action sociale : nous voulons parler des lieux d'accueil où, au quotidien, se rencontrent concrètement des publics en demande d'aide et des intervenants qui, soit parce que c'est leur métier, soit parce qu'ils ont fait le choix de s'y consacrer bénévolement, font face à ces attentes.

C'est aux pratiques développées dans ces lieux d'accueil qu'est consacré cet article et, plus précisément, aux difficultés et aux enjeux soulevés, du côté des accueillants, par le contact avec les demandeurs d'asile. Comme nous essaierons de le montrer, si l'émergence de cette nouvelle figure de l'exclusion sociale pose problème ${ }^{1}$, ce n'est pas qu'en raison de la charge qu'elle fait mécaniquement peser sur une offre de prestations dont les ressources (matérielles, humaines) sont limitées. C'est aussi parce que, à bien des égards, les demandeurs d'asile apparaissent comme des publics atypiques, qui, de par leur parcours, mais aussi de par le statut, interrogent les fondements mêmes du travail social comme pratique professionnelle, et au-delà, la relation d'aide comme forme de lien interpersonnel.

\footnotetext{
1 L'usage que nous faisons ici de la notion de problème est à double sens. Le premier renvoie aux contraintes objectives que fait peser sur les services l'accroissement quantitatif des attentes formulées à leur égard. Mais l'accueil des demandeurs d'asile n'est pas qu'un problème qui se pose de l'extérieur aux acteurs locaux ; c'est aussi un problème que l'on pose au sens où, à partir d'une réalité démographique qui s'impose aux acteurs, des logiques sociales (institutionnelles, professionnelles, etc.) se déploient qui concourent à configurer ce qui, dès lors, est à appréhender comme un problème d'action publique locale. Dans cette seconde acception, la question n'est plus tant de savoir s'il est vrai qu'il y a problème, ou pourquoi il y a problème, que de comprendre sous quelle forme émerge ce qu'une configuration d'acteurs considère comme un problème et à saisir les mécanismes de cette construction sociale.
} 


\section{Encadré 2 : Méthodologie de l'enquête}

L'enquête sur laquelle nous nous appuyons a été réalisée en 2003 dans le cadre d'un programme de la DREES portant sur l'accueil des étrangers dans les dispositifs d'hébergement généralistes et spécialisés.

L'étude, menée dans les Alpes-Maritimes, comprenait quatre axes:

- la description de l'offre locale de prise en charge et des conditions effectives d'hébergement des demandeurs d'asile, réfugiés et déboutés du droit d'asile ;

- l'étude des caractéristiques des populations concernées en termes de profils et de trajectoires ;

- la conduite d'une enquête exploratoire consacrée aux méthodes d'investigation envisageables en vue d'une meilleure connaissance, à grande échelle, des publics accueillis ;

- l'analyse de la politique locale menée en matière d'hébergement.

Dans le présent article, nous nous appuyons principalement sur le versant de l'enquête consacré à l'analyse de la politique locale.

Trente-huit entretiens ont été réalisés auprès de membres d'institutions et de structures concernées par cette thématique. (Cette étude a donné lieu à un rapport (Frigoli, Jannot, 2004)). Dans le cadre de ce programme, une autre équipe a travaillé sur le même thème dans le département du Rhône (Bourgeois, Ebermeyer, Sevin, 2004).

\section{- L'accueil des demandeurs d'asile dans les Alpes-Maritimes : éléments de contexte et politique locale}

C'est de manière très soudaine que l'accueil des demandeurs d'asile est devenu dans les Alpes-Maritimes un objet de préoccupations pour les autorités publiques, notamment les institutions en charge des populations migrantes et, au-delà, pour l'ensemble des acteurs concernés par le sort des personnes en difficulté. Au cours de l'année 2000, la préfecture enregistra une hausse importante, autant qu'imprévue, du nombre de demandes : on passe ainsi de 283 demandes d'asile conventionnel en 1999 à 833 en 2000, puis à 904 en 2001 ; cette augmentation touche pendant la même période l'asile territorial qui passe entre 1999 et 2001 de 416 à 950 demandes ${ }^{1}$.

Dès les premiers signes de ce que beaucoup décrivent localement comme un " afflux massif », la pression exercée sur l'offre locale d'hébergement est forte. Ce sont, chaque semaine, des dizaines de personnes isolées et de familles qui se présentent aux différents guichets d'accueil susceptibles de leur fournir un toit : Service social d'aide aux émigrants (SSAE), lieux d'accueil caritatifs, services du Schéma départemental d'urgence (SDU),

1 Source : préfecture des Alpes-Maritimes. 
centres communaux d'action sociale (CCAS), polyvalence de secteur, directions départementales des Affaires sanitaires et sociales (DDASS), etc. Rapidement, des procédures de coopération entre les services de l'État et les acteurs de terrain qui font face à ces demandes, se mettent en place en vue d'utiliser au mieux le dispositif d'hébergement spécialisé départemental (CADA et $\mathrm{CPH}$ ). Mais celui-ci, qui totalise 101 places, est très vite saturé et, face à des demandes d'hébergement qui se font de plus en plus pressantes, c'est l'ensemble de l'architecture de l'offre locale d'hébergement d'urgence qui se trouve mise à contribution : accueil de nuit, CHRS, centre maternel et, surtout, hôtels meublés dans lesquels vont se trouver majoritairement hébergés les demandeurs d'asile. Dans le cadre d'une opération de recensement des publics accueillis dans les structures d'hébergement « un jour donné », nous avons pu établir, qu'en novembre 2003, $60 \%$ des publics tous statuts confondus (demandeurs d'asile, réfugiés, déboutés) étaient hébergés à l'hôtel (Frigoli, Jannot, 2004).

Mais les difficultés ne concernent pas que le logement. Les acteurs de terrain découvrent une population qui, outre le fait qu'elle se présente en masse aux guichets de l'action sociale, témoigne de difficultés et exprime des demandes qui relèvent manifestement de ce que certains travailleurs sociaux nomment la " grande urgence » : l'état physique des personnes, dont des femmes enceintes, est préoccupant. Les familles sont nombreuses, des enfants, parfois des nourrissons, présentent des carences de toutes sortes. Beaucoup, dans un mauvais français, évoquent les conditions effroyables qu'ils ont dû affronter lors de leur parcours. De ce fait, les demandes formulées relèvent, outre la recherche d'un logement, de ce que l'on qualifie généralement dans le secteur social de « besoins primaires » : nourriture, vêtements, premiers soins. S'y ajoute l'attente d'une domiciliation, préalable nécessaire à l'inscription dans un parcours dont les bénévoles associatifs, les travailleurs sociaux généralistes, sont loin de toujours connaître le cadre juridique.

Du côté des associations, caritatives notamment, on se trouve en effet soudainement confronté à des publics inhabituels, dont on ne sait rien du parcours et des projets, avec lesquels il est difficile de communiquer, dont la situation au regard de la législation est incertaine, et dont on ne connaît guère les droits. Dans un contexte où les routines, les « réflexes » professionnels ou forgés par l'expérience de l'accueil d'urgence se trouvent en partie inopérants, et en l'absence de crédits spécifiques, on essaie donc de faire face à l'urgence : on mobilise son carnet d'adresse, on alerte sa hiérarchie, on essaie d'obtenir des informations concernant les possibilités concrètes d'action et le cadre juridique dont celles-ci relèvent. On tente de se forger une compétence, on essaie de se donner des principes d'action, voire des principes de justice, face à une situation dans laquelle, faute de moyens, il faut faire des choix.

Les acteurs associatifs généralistes sont en première ligne et véritablement désemparés, mais du côté des acteurs officiellement en charge des 
populations migrantes, ou des administrations qui ont la responsabilité de dispositifs d'aide en faveur des publics en difficulté, le désarroi n'est pas moindre. Le SSAE est submergé et contraint de mettre temporairement entre parenthèses certaines de ses missions habituelles. Les services de la préfecture tentent, non sans mal, d'endiguer un flux de migrants qu'ils préféreraient voir se diriger vers d'autres départements. La DDASS fait face à des problèmes de financement des nuits en hôtel meublé notamment, mais aussi à des contraintes organisationnelles fortes : il faut favoriser l'adaptation d'une configuration d'acteurs préexistante au sein de laquelle l'administration sociale est placée en position d' " animateur», tout en la préservant d'une déstabilisation trop brutale. De plus, au souci de maintenir de bonnes relations avec ses partenaires habituels, s'ajoute pour la DDASS la volonté d'éviter que l'attention portée aux publics traditionnels de l'urgence sociale diminue alors que du mécontentement à ce sujet commence à se manifester.

De fait, il faudra attendre l'été 2001 pour que se mette en place un dispositif d'hébergement exclusivement consacré aux demandeurs d'asile, INTERMED, qui est un dispositif consacré exclusivement à l'hébergement transitoire des demandeurs d'asile en meublé ou à l'hôtel. Il est géré par l'association qui avait jusque-là, en charge le Service départemental d'urgence. Puis en septembre 2003 sera créé un lieu dédié à l'accueil et l'orientation de ce type de public : la plateforme d'accueil des demandeurs d'asile. Gérée par la même association, elle a vocation à faire office de guichet unique pour les demandeurs d'asile en assurant accueil, domiciliation, orientation vers les structures pertinentes.

Entre cette première phase, courant 2000, marquée par l'urgence et le « bricolage » institutionnel, et la mise en place de ces deux outils, les pratiques d'accueil et de prise en charge puiseront aux régulations d'un système d'action local confronté à de fortes turbulences et soumis à l'introduction d'un nouvel enjeu de coopération. Et ce, à travers le double mouvement que décrit Borraz (1999) lorsqu'un « nouveau problème » fait irruption dans le paysage de l'action publique locale : les problèmes sont définis en fonction des cadres organisationnels (les modes de coopération) et cognitifs (les manières d'envisager les problèmes) existants ; mais ces cadres s'adaptent aux problèmes en fonction des enjeux que suscite chez les acteurs concernés la perspective d'avoir à gérer ces problèmes en commun. Les choix et les politiques organisationnels seront à ce titre divers. Ainsi, nous avons pu identifier plusieurs logiques d'adaptation organisationnelle à l'émergence de ces nouveaux publics que constituent les demandeurs d'asile, notamment :

- une posture défensive adoptée par certaines structures souhaitant se préserver face à des publics dont le statut particulier rend incertaine toute démarche centrée sur l'insertion sociale et professionnelle (les demandeurs d'asile étant des personnes dont par principe on ne sait pas de quoi l'avenir - subordonné à l'obtention du statut de réfugié - sera fait); 
- une logique de spécialisation adoptée par des structures associatives engagées sur la voie de la constitution d'une expertise en la matière ;

- une démarche de bricolage institutionnel développée par des organisations enrôlées nolens volens dans le réseau d'accueil et recherchant l'équilibre entre une adaptation nécessaire et la volonté de ne pas trop dévier de leur « sentier institutionnel ».

Toutefois, quelles que soient les postures adoptées par les structures d'accueil, celles-ci auront en commun de faire pour une large part reposer sur les acteurs - qui font face au quotidien aux publics et à leurs demandes la politique locale développée à l'égard des demandeurs d'asile. Cette dernière ne se réduit pas aux pratiques mises en œuvre dans les divers lieux d'accueil et le sort des publics n'est pas entièrement contenu dans les dynamiques d' " échanges au guichet », mais, au quotidien, la survie sociale des demandeurs d'asile passe pour une large part par ce type d'échanges ${ }^{1}$. De ce fait, nombreux sont ceux qui, parmi les travailleurs sociaux et les bénévoles associatifs, vont devoir faire avec les demandes d'une population qui, jusque-là, leur était inconnue, ce qui nous conduit à faire l'hypothèse d'une déstabilisation des modèles d'intervention sociale.

\section{- Le demandeur d'asile au guichet}

Les difficultés posées par l'accroissement des demandes d'hébergement, et au-delà, des demandes d'aide alimentaire, de soins ou d'aide financière, furent en premier lieu d'ordre matériel. L'arrivée des demandeurs d'asile apparut comme une charge nouvelle pesant sur une offre de prestations, au sens large, et sur des ressources financières et humaines qui trouvèrent rapidement leurs limites. En clair, c'est avant tout d'un manque de solutions concrètes à apporter aux demandes de ces publics " surnuméraires » dont souffrirent les diverses structures : hébergement comme nous l'avons vu ${ }^{2}$, mais, plus largement, dans tous les domaines que couvre le secteur de l'action sociale d'urgence : nourriture, vêtements, soins, bons de transport, bons de vacances pour les enfants, etc. Les moyens financiers vinrent rapidement à manquer, notamment au sein d'associations qui, fonctionnant sur avance de trésorerie en matière d'hébergement, eurent à faire face au mécontentement, voire à la colère de gérants d'hôtels meublés victimes de retards de paiement qui, en raison des prix pratiqués, proportionnels aux

1 C'est ce que montre notamment le travail qu'Evelyna Dargyte (2002) a consacré aux pratiques quotidiennes des migrants de l'ex-URSS sur la Côte d'Azur.

2 Outre l'insuffisance de places en CADA, le département des Alpes-Maritimes souffre à la fois d'un déficit de logements sociaux et d'une vocation touristique qui alimente l'inflation des loyers et des tarifs pratiqués au sein du parc hôtelier. 
loyers à Nice, atteignirent assez vite des sommes importantes ${ }^{1}$. Les moyens humains disponibles tant au sein des services de l'Etat ou des services sociaux de la municipalité, qu'au sein des associations locales furent également insuffisants. En bref, ce fut d'abord sur le plan logistique que l'arrivée des demandeurs d'asile « fit problème ».

Mais cette réalité comptable, quel qu'en soit le poids, ne saurait masquer le fait qu'avec les demandeurs d'asile, on s'est trouvé localement confronté à de nouveaux publics, en tout cas appréhendés comme tels par beaucoup d'intervenants sociaux. De plus, il semble que, de par certaines caractéristiques qui lui sont attribuées, le demandeur d'asile ait été également appréhendé (et le soit encore dans une large mesure au moment où nous écrivons ces lignes) comme un usager atypique, mettant par là en lumière, à travers les habitudes, les routines, les « allant de soi » qu'il brise, quelques-uns des éléments stables de l'univers familier des travailleurs sociaux et des bénévoles qui accueillent quotidiennement les personnes marginalisées.

\section{Le statut au regard de la législation : "Mais à quoi ont-ils droit?" "2}

Parmi les éléments qui suscitèrent la perplexité de nombre d'intervenants sociaux, il faut compter l'absence d'informations juridiques sur le statut des demandeurs d'asile et, de ce fait sur les droits auxquels ils peuvent prétendre. Certes, au Secours catholique, au Secours populaire ou au sein de l'Entraide protestante, il n'est pas rare d'avoir affaire à des personnes étrangères ou « issues de l'immigration ». Mais la catégorisation des publics, et donc leurs droits, sont généralement sans ambiguïté et les critères correspondants sont connus par la plupart des acteurs : soit les personnes sont de nationalité française et éligibles de plein droit aux différents droits sociaux; soit les personnes sont étrangères et en situation régulière et peuvent accéder aux prestations correspondant à leur titre de séjour; soit, enfin, les personnes sont en situation irrégulière et il reste aux intervenants sociaux à voir dans quelle mesure ils souhaitent mobiliser les prestations d'assistance qu'ils ont à leur disposition.

Avec les demandeurs d'asile, la situation est tout autre. Les acteurs de terrain se sont trouvés soudainement face à des publics étrangers, majoritairement non francophones, parfois en situation irrégulière au moment de la rencontre mais désireux de s'inscrire, ou déjà inscrits, dans un parcours juridique dont

$\overline{1}$ Le montant de la location d'une chambre en hôtel meublé à Nice peut s'élever jusqu'à 2000 euros par mois. Parmi les associations qui eurent fréquemment recours à ce type d'hébergement (avec accord de la DDASS mais sur avance de trésorerie) beaucoup se trouvèrent en quelques mois dans une situation financière très précaire du fait de remboursements, venant de l'État, qui tardèrent à leur être versés. C'est une des raisons qui conduisit l'une d'elle, fortement impliquée jusque-là, à mettre un terme à ce type de pratiques en mars 2001.

2 Les éléments de sous-titre entre guillemets sont extraits d'entretiens que nous avons menés. 
les subtilités échappaient à beaucoup. À partir de quelle étape de la procédure la personne peut-elle être considérée comme légalement admise à séjourner sur le territoire ? Pour combien de temps ? Quels sont les droits correspondants? Les choses se compliquèrent encore lorsque apparurent les premiers déboutés du droit d'asile puis ceux que, localement, on nomme les « déboutés de déboutés " ${ }^{1}$. Au fil des semaines, les situations individuelles et familiales se complexifiant, la socialisation aux règles et procédures de la demande d'asile, - ainsi qu'aux usages qu'il est possible d'en faire s'imposa et favorisa parmi les intervenants sociaux les plus motivés par cette quête permanente de la bonne information ou des "procédures qui marchent » l'émergence de « spécialistes » locaux en la matière.

Si le demandeur d'asile apparut initialement comme une sorte d' « ovni » parmi les usagers de l'action sociale, ce fut d'abord sur le plan juridique. C'est, avec cette incertitude concernant ce que la loi autorise en termes de pratiques d'intervention sociale, que les acteurs de terrain se sont vus fragilisés, qu'il s'agisse de travailleurs sociaux diplômés pour qui la connaissance de la législation est une ressource professionnelle essentielle, ou de bénévoles associatifs tout simplement désireux de bien faire.

\section{Le passage obligé par l'assistance : "c'est pas du travail social»}

Mais la singularité du statut des demandeurs d'asile au regard de la législation eut une autre incidence sur les pratiques d'accueil et de suivi social : dans une large mesure, ce statut ne laisse d'autre choix que de se tourner vers un mode d'intervention sociale, l'assistance, qui n'a pas une bonne image dans le travail social professionnalisé. Ici, la notion d'assistance s'oppose moins à celle d'assurance, comme c'est généralement le cas dans les classifications officielles, qu'à celle de projet. Autrement dit, dans le monde du travail social, relève de l'assistance toute pratique visant à combler un besoin avéré et à s'en tenir là. La connotation péjorative procède précisément de cette accointance avec la logique humanitaire appréhendée, sur le modèle de l'urgence médicale, comme une réponse ponctuelle à des besoins vitaux et qui se conjugue au présent (Sassier, 1997). Or, avec les demandeurs d'asile, il est par principe difficile d'inscrire l'action dans la durée : d'abord parce que les prestations légales et les outils du processus d' « autonomisation de la personne » cher aux assistantes sociales (Revenu minimum d'insertion (RMI), Fonds d'aide aux jeunes (FAJ), etc.) font défaut dans ce cas. Mais, plus fondamentalement, c'est l'ambiguïté de la situation sociale des demandeurs d'asile, nous y reviendrons, qui est en cause. Soumis à la décision de l'OFPRA ou du ministère de l'Intérieur, l'avenir des demandeurs d'asile est par principe incertain, de même que la

1 Il s'agit de personnes qui ont introduit sans succès un recours. 
durée de l'incertitude elle-même. Dans ces conditions, la maxime qu'aiment à reprendre de nombreux travailleurs sociaux, "apprendre à pécher plutôt que donner du poisson » est en partie vidée de sa substance. Et on comprend que ce soit sans grand enthousiasme qu'ils se prêtent à des pratiques de type caritatif (fourniture de nourriture, de vêtements, etc.) qui heurtent les principes de professionnels formés à l'exercice d'un travail social « émancipateur ».

\section{Travail social versus militantisme : "Je ne suis pas responsable de la politique de l'État français"}

Mais c'est aussi pour une autre raison que, pour certains, l'action auprès des demandeurs d'asile, "ce n'est pas du travail social " ${ }^{1}$. Nous voulons parler des rapports qu'entretiennent, dans le cas de l'action auprès de ce type de public, le monde du travail social, du bénévolat, et du militantisme. Non pas que la question ne se pose pas dans d'autres domaines d'intervention sociale. La tension entre, d'un côté, la froide distance affichée à l'égard des populations par une conception du travail social comme pratique d'expert et, de l'autre, la compassion du bénévole, la passion du militant, parcourt toute l'histoire du travail social. Et on sait l'énergie qu'ont déployée certains groupes professionnels pour s'émanciper du modèle de la «dame patronnesse », prendre leurs distances avec la posture militante, afin de promouvoir l'image d'un véritable corps professionnel, légitimé par des savoirs et des techniques spécifiques (voir par exemple le rôle joué par 1'Association nationale des assistants de service social (ANAS) dans les années soixante tel que le décrit Mondolfo, (1997)). Bref, le caractère équivoque de l'attitude qui consiste à être aux côtés de ceux dont on s'occupe ne constitue pas un fait nouveau en soi.

En effet, la question des limites et de la nature de l'engagement qu'il convient de manifester face à la détresse de l'autre se pose au quotidien dans de très nombreuses situations d'interaction avec les demandeurs d'asile. Par exemple, faut-il ou non intervenir dans le cadre de la procédure OFPRA ? Une chose est d'aider une personne à survivre socialement, une autre est de tenter de contribuer à faire d'elle un réfugié politique. On peut raisonnablement supposer que l'objectif de tout travailleur social est de faire sortir les usagers qu'il côtoie de leurs difficultés. Ici peuvent intervenir et se mêler, selon les acteurs et les situations, des techniques professionnelles, des sentiments d'empathie et, éventuellement, une vision critique des politiques publiques menées en la matière. Mais, avec le demandeur d'asile, il est aussi question de droits de l'homme à défendre. (Non pas qu'il n'en soit pas question en matière de lutte contre l'exclusion). Dans un

1 C'est bien ce que signifie une assistante sociale : "Il n'y a que le caritatif qui a la souplesse nécessaire. Mais on ne peut pas inscrire notre action dans la durée. C'est pas du travail social». 
contexte où circulent des discours explicitement hostiles à l'immigration d'une part et où l'OFPRA reconnaît à moins de $10 \%$ des demandeurs le statut de réfugié d'autre part, il est difficile de se soustraire et ce peut-être davantage que face aux Rmiste ou aux travailleurs pauvres, à l'obligation de prendre position dans leurs pratiques les plus quotidiennes. Aider un demandeur d'asile à devenir un réfugié peut être envisagé comme un acte professionnel visant à permettre « l'accès à l'autonomie de la personne »; comme un acte humanitaire s'inscrivant dans un «devoir de solidarité » que l'on s'impose ; mais cela peut aussi être perçu comme le signe d'un engagement outrepassant le mandat qui est officiellement confié aux intervenants sociaux. Or ce n'est pas toujours ce que souhaitent, ou ce que pensent possible, des travailleurs sociaux soumis au cadre normatif des politiques publiques dans le cadre desquelles ils interviennent alors même qu'ils sont parallèlement interpellés par des militants associatifs n'hésitant pas à pointer du doigt le manque d'engagement de certains professionnels ${ }^{1}$.

\section{La différence culturelle : "cela veut dire quoi dans sa culture?"}

Dans les entretiens que nous avons réalisés, les intervenants sociaux, quel que soit leur statut, font souvent référence à la "différence culturelle » qui les sépare des demandeurs d'asile en la présentant comme une des difficultés qu'ils doivent affronter. Cette problématique, qui n'est pas propre à la demande d'asile (Guelamine, 2001), semble prendre un relief particulier dans un contexte où la maîtrise du français est rare. C'est en effet, en premier lieu, la question de la langue qui est évoquée. Outre les problèmes liés à l'obtention de renseignements de type administratif, le défaut de compréhension mutuelle serait préjudiciable à une certaine conception du travail social en tant que pratique fondée sur l'échange de parole et l'intersubjectivité. Le recours aux concepts et aux méthodes de la psychologie clinique, qui constitue pour beaucoup le cadre paradigmatique de la relation d'aide, s'avérerait alors en partie inopérant. Cette importance donnée à la maîtrise de la langue française n'est pas dans certains cas sans incidence sur les pratiques de sélection des candidats à l'entrée dans une structure d'aide : "Nous, on travaille sur un contrat relationnel qui passe par la parole. Et c'est dur de le faire avec des paroles sommaires. On n'a pas les moyens d'avoir un interprète lors de chaque entretien. Donc on est réticent face aux familles qui ne parlent pas français. Cela peut paraître archaïque comme argument mais d'un autre côté dans des structures où on ne peut être efficace que si il y a un contact, on se sent moins efficace [...]. Quand on

$\overline{1}$ On pourrait illustrer la même idée par le cas des « sans papiers » qui confronte les travailleurs sociaux à l'obligation de se positionner - en tout cas à ce qui peut être perçu comme un positionnement - face à l'illégalité de la situation dans laquelle se trouvent alors les personnes accueillies. 
présente les familles, on demande tout de suite si quelqu'un parle français dans la famille [...]. Quand c'est clair qu'on ne va pas se comprendre, ça fait barrage. On est confronté à des barrages où en fin de prise en charge on ne saura toujours pas si on s'est compris [...] Tant qu'à faire, on se dit qu'avec une autre famille, on va être efficace en six mois. D'où une certaine frilosité face aux demandeurs d'asile ». (Intervenant associatif).

Mais, au-delà de la difficulté à s'exprimer en français, nombreux sont ceux qui, parmi les intervenants sociaux, considèrent l'appartenance des demandeurs d'asile à une « autre culture » comme un frein s'opposant à la compréhension des situations individuelles et familiales. Au premier rang des domaines de la vie sociale qui justifient l'adoption d'un regard ethnicisé sur les demandeurs d'asile, se situent les pratiques éducatives. Les ressortissants des « pays de l'Est » notamment - les demandeurs d'asile à Nice sont majoritairement de Tchétchénie ${ }^{1}$ - auraient ainsi une approche de l'éducation très différente des principes qui fondent l'éducation " à la française ». On peut à ce sujet citer les propos suivants de divers intervenants : "les enfants ne sont pas élevés de la même façon. Ils n'ont pas les mêmes références éducatives "; ou " ce sont des gens qui vivent peut-être un peu différemment en termes de culture parce que les enfants par exemple sont moins surveillés, ils vivent plus en communauté, ils ont des habitudes communautaires beaucoup plus fortes »; ou encore "leur mode de vie n'a rien à voir avec le nôtre. Ils laissent leurs enfants jouer dehors jusqu'à onze heures du soir ».

Mais l'attribution de traits culturels comme éléments d'explication des comportements observés est un processus que l'on retrouve dans de très nombreuses situations, dès lors que les intervenants font face à des conduites incompréhensibles au regard des cadres interprétatifs habituels. On peut citer à ce sujet les propos de cet intervenant associatif pour qui « si il y a la confiance, on s'en sort toujours. Mais si on sent que dans les choses qui ne sont pas dites il n'y a pas seulement un manque de vocabulaire [...], alors face à ça c'est quoi ? c'est qu'il ne parle pas bien? Celui qui rigole à tout ce qu'on dit, ça veut dire quoi ? Qu'il a tout compris et qu'il se moque de nous? C'est une manière polie de dire: "Je vous en dirai pas plus"? Cela veut dire quoi dans sa culture? S'il parle français, on peut toujours creuser. Sinon, c'est quoi le problème? C'est qu'il ne comprend pas? C'est qu'il se bloque dans sa langue de peur de se livrer?".

Parfois, la référence à l'origine culturelle des migrants apparaît comme une manière pertinente de distinguer les individus en fonction de leur groupe d'appartenance : " ceux de l'Est sont encore dans une culture d'assistanat »; ou "les personnes de l'Est ont une énergie, une foi dans l'avenir, un courage, c'est fantastique, c'est tout l'opposé des autres ».

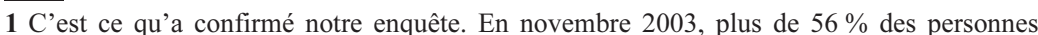
accueillies dans les structures d'hébergement spécialisées et généralistes des Alpes-Maritimes étaient russes, la très grande majorité d'entre elles se déclarant originaires de Tchétchénie. Voir Frigoli, Jannot (2004). 
Le recours à des formations spécialisées dans le domaine de «l'interculturel » constitue une des manières de s'adapter à des pratiques perçues comme relevant de la culture (faire la manche par exemple).

On voit également la trace de cette perception dans des pratiques telles que la recherche de la " mixité » dans la répartition des populations sur le territoire de la ville ou dans certaines structures, démarche visant de manière volontariste à contrer la tentation du " repli communautaire ».

\section{Le statut social dans le pays d'origine : "des gens cultivés, qui ont de l'enseignement"}

Dans le discours des intervenants sociaux, il est par ailleurs souvent fait référence au « statut social élevé » que, selon eux, occupaient nombre de demandeurs d'asile dans leur pays d'origine ${ }^{1}$. Derrière cette notion se mêlent différents éléments reliés à ce que l'on sait, ou suppose, de la carrière scolaire de la personne, du métier qu'elle exerçait avant la migration, des responsabilités qu'elle assumait, bref des ressources dont elle était apparemment détentrice dans les différentes sortes de capitaux (social, économique, culturel) qu'il est d'usage de considérer comme allant de pair avec l'appartenance aux couches sociales supérieures. Or, il s'agit là de profils assez inhabituels pour des travailleurs sociaux et des bénévoles que l'expérience de l'action sociale d'urgence amène davantage à fréquenter des publics issus des classes populaires. Et il semblerait que, pour certains, cela soit vécu comme déstabilisant de se trouver confronté à d'anciens "ingénieurs ", "médecins », "chefs d'entreprise ", "hauts fonctionnaires ", qui sont eux-mêmes réticents à devenir le « client » d'une assistante sociale ou aux méthodes classiques de l'entretien d'aide. Face à ce type de public, l'inconfort ressenti par certains travailleurs sociaux peut être lié à une attitude de refus pur et simple par l'usager de toute approche " psychologisante » de la relation d'aide : "très souvent, les personnes sont des personnes qui avaient un statut social chez elles qui leur permet de se passer de travailleur social. Ils nous disent: "mais nous, on n'a pas besoin de travailleur social; donnez-moi un travail. On demande telle aide mais pour le reste, nos enfants, on sait les élever, le français on sait l'apprendre". Dans ce cas, ils choisissent une voie plus aride mais plus autonome ». (Intervenant associatif).

Mais sont également parfois évoquées des situations qui attestent d'une méconnaissance des modes de fonctionnement institués du travail social, c'est-à-dire des principes qui, traditionnellement, organisent la relation entre un travailleur social et un usager : par exemple le principe selon lequel dans cette relation, " il y a celui qui sait et celui qui ne sait pas, celui qui est

1 Il ne nous a pas été possible de confirmer statistiquement cette idée, ne serait-ce que parce que les informations liées à la profession exercée sont le plus souvent difficilement exploitables. Rappelons toutefois que notre propos n'est pas ici de confirmer ou non la véracité des discours tenus par les acteurs de terrain mais de les recueillir pour ce qu'ils révèlent de la manière dont les demandeurs d'asile, en tant qu'usagers des services sociaux, sont appréhendés localement. 
inséré et celui qui ne l'est pas ». Dans ce cas, on se trouve face à un désaccord implicite concernant la «définition de la situation », le demandeur refusant de confirmer la répartition des rôles et des attentes qui va de pair avec la définition que les « professions », au sens anglo-saxon du terme, sont enclines à instaurer avec leurs « clients », soit une définition qui fixe une séparation nette entre un expert détenteur de solutions et un demandeur en attente... de ces mêmes solutions : "Là, on a des gens qui étaient dans la bonne société. On avait découvert ça quand on a reçu les Kosovars. On a eu un maire, un ingénieur qui avait 600 personnes sous ses ordres. On a un renversement des valeurs... ". C'est un des fondements mêmes du travail social comme pratique de re-socialisation qui est alors remis en cause par des individus qui ne se reconnaissent pas comme désocialisés.

\section{Le projet migratoire : "ceux qui viennent simplement pour utiliser le système "}

Il est enfin, parmi ceux que nous avons repérés dans le discours des acteurs de terrain, un dernier élément propre aux demandeurs d'asile et agissant dans le sens d'une déstabilisation des pratiques d'intervention sociale. Celui-ci concerne le projet migratoire que l'on attribue à ces derniers. Non pas, loin de là, que les acteurs rencontrés reprennent explicitement à leur compte le clivage qui sépare les « vrais » des « faux » réfugiés et qu'ils aient pour souci principal et systématique d'essayer de situer les individus des deux côtés de cette frontière. Simplement, il est dans la logique de la relation d'aide d'essayer de comprendre la situation sociale, le parcours des personnes que l'on rencontre, et lorsqu'il s'agit de migrants, de reconstituer le contexte dans lequel ceux-ci sont venus en France.

Les typologies de parcours et de projets migratoires que dressent les acteurs sont diverses, de même que le lexique utilisé pour les décliner. C'est l'image d'un continuum qui nous semble le mieux rendre compte de la variété des figures de la migration que distinguent les intervenants sociaux. À un extrême prend place celle du " passeur », personnage mafieux dont chacun reconnaît et dénonce l'existence dans le monde de la demande d'asile. À l'autre, se situe la victime indiscutable, personnage tragique de par les épreuves, extrêmement choquantes même pour des acteurs rompus au contact avec la misère, qu'elle a traversées. Entre les deux, on trouve l'ensemble de ceux qui ne méritent ni la condamnation qui frappe les premiers, ni la compassion que s'attirent les seconds. Il s'agit de ces personnes qu' on n'a aucune raison de ranger du côté des " salauds qui font de l'argent sur la misère des autres ", mais dont les motifs et les conditions de la migration ne sont pas totalement clairs.

Le problème pour les intervenants sociaux n'est pas tant, en soi, de devoir faire avec cette incertitude concernant le projet migratoire des migrants. Cet élément de caractérisation des publics est loin d'être le ressort essentiel 
des pratiques que développent des acteurs de terrain qui, répétons-le, n’ont pas l'obsession du tri entre les « vrais » et les « faux » réfugiés. Il n'est pas non plus de devoir s'accommoder de l'idée que les usagers qu'ils rencontrent puissent avoir des comportements « stratégiques » dans l'exercice de présentation de soi qui leur est fréquemment imposé : c'est le lot commun de tous ceux qui se donnent pour projet professionnel ou personnel d'aider les personnes en difficulté que de découvrir, tôt ou tard, qu'il n'y a aucune raison de leur refuser le statut d'acteur au sens plein du terme, même si les compétences correspondantes sont inégalement distribuées.

En fait, ce qui pose problème, c'est davantage le caractère particulièrement marqué de l'opposition entre deux figures, celle du mafieux et celle du persécuté, qui coexistent au sein du même public. En effet les enjeux des choix que l'on fait et de la posture que l'on adopte face au discours de l'autre sont singulièrement alourdis par le caractère tragique des situations telles qu'elles sont racontées : si la personne dit vrai, alors, ne pas la croire, ou simplement faire preuve de circonspection à l'égard de son propos apparaît comme moralement insupportable. Décider d'établir un écart systématique, fût-ce pour s'épargner la déconvenue d'avoir été dupé, entre la souffrance telle qu'elle est racontée et telle qu'elle est vécue, c'est prendre le risque d'ajouter une injustice de plus à un parcours qui en est parsemé. Peut-on instaurer la prudence, le doute, comme position de principe face à des drames (viols, torture...) d'une telle intensité ? La difficulté, on le comprend, est dans la recherche de la « bonne distance » vis-à-vis des personnes et de ce qu'elles racontent.

Certes, il n'y a rien de fondamentalement nouveau dans cette recherche du juste équilibre entre ce que d'aucuns considéreraient comme de la naïveté et, à l'opposé, la méfiance systématique à l'égard du discours des usagers. C'est une des figures imposées du travail social que d'être capable de ne pas s'apitoyer sans pour autant être indifférent, d'accorder sa confiance à l'autre tout en se préservant des désillusions que peut occasionner une candeur excessive.

Mais il est singulièrement difficile de le faire dans le cas de l'accueil des demandeurs d'asile face à leurs récits qui décrivent une détresse sociale extrême et au commerce de la misère ${ }^{1}$; face au rôle que joue la capacité à

\footnotetext{
1 Nous faisons référence aux nombreuses anecdotes que nous ont livrées nos interlocuteurs quant à l'existence de pratiques consistant, de la part d'individus qui font commerce de la misère dans laquelle se trouvent les demandeurs d'asile, à monnayer l'accès à certaines informations utiles, voire indispensables à leur survie. Ainsi en est-il, pour ne donner que cet exemple, de la « tarification » de l'accès à certaines adresses ou personnes du secteur caritatif, qui, à leur insu, deviennent ainsi l'objet d'un commerce dont elles sont les premières à dénoncer l'existence. Notre enquête, comme notre propos ici, n'a pas vocation à attester de l'existence de pratiques dont l'évocation obéit en partie aux mécanismes de diffusion de la rumeur. Toujours est-il que, quels que soient les faits objectifs qui l'alimentent, ce discours participe d'un mode d'appréhension de la demande d'asile qui en fait un monde dur, en premier lieu pour les demandeurs d'asile eux-mêmes, ce qui ne contribue pas peu à susciter l'embarras d'intervenants sociaux qui se voient confrontés à un univers dans lequel se côtoient la misère la plus extrême et, du côté des «passeurs », le cynisme le plus impitoyable.
} 
se raconter dans leur sort tant auprès de la préfecture qu'à l'OFPRA en passant par l'entrée en CADA. À cela s'ajoute le fait qu'au final plus de $80 \%$ des demandeurs en France n'obtiendront pas le statut de réfugié. Quelles que soient les raisons qui justifient ce chiffre, il contribue à faire circuler un doute quant à la véracité des récits que produisent les demandeurs d'asile.

\section{- De l'ambiguité du statut des demandeurs d'asile à l'ambivalence du regard porté sur cette catégorie d'usagers}

L'ensemble des éléments qui précèdent peut sans doute expliquer que, pour le moins, l'accueil et le suivi des demandeurs d'asile, en tant que nouveaux usagers de l'action sociale d'urgence, ne soient pas perçus comme chose aisée par beaucoup d'intervenants sociaux. En témoigne la relative ambivalence que nous avons cru déceler dans nombre de propos que nous avons recueillis au sujet de ces migrants que l'on plaint mais dont on dit que "beaucoup ne sont pas faciles"; de ces demandeurs d'asile dont on déplore la vie précaire mais qui, pour certains d'entre eux, " deviennent rapidement exigeants »; de ces familles qui manquent de tout mais dont certaines « savent très bien utiliser les dispositifs d'aide »...

Qu'on ne s'y trompe pas toutefois. Ce type de discours renvoie moins aux caractéristiques comportementales de cette catégorie d'usagers qu'il ne traduit l'embarras des intervenants sociaux face à leur statut pour les raisons examinées plus haut.; il est surtout déstabilisant pour eux de se trouver confrontés à des personnes dont le statut oscille entre celui de victime d'un système (celui qu'elles disent avoir fui) et celui de coupable d'en détourner un autre (celui auquel elles demandent protection). Que sont donc les demandeurs d'asile pendant les mois que dure la procédure sinon des personnes qui, tels ces justiciables placés en détention préventive, sont présumées de bonne foi jusqu'au jour du jugement mais enfermées dans une situation qui leur interdit, au nom d'un principe de précaution, de mener une vie normale? Le législateur a prévu, d'un côté, des mesures de bienveillance que doivent par principe manifester les États démocratiques à l'égard des opprimés (DNA, aides financières spécifiques, scolarisation des enfants, possibilité de recours, etc.), de l'autre, des restrictions (interdiction de travailler, limitation des droits sociaux accessibles, recours à la notion de « pays sûr », etc.) qui manifestent la volonté de ne pas banaliser la demande d'asile dans le champ de la migration et de maîtriser les flux migratoires. Mais s'il est possible de justifier politiquement et donc d'assumer sans grande difficulté cette posture, il en va tout autrement pour ceux qui doivent au quotidien dans les lieux d'accueil d'urgence faire tenir ensemble les deux faces d'une figure double. C'est bien en effet sous ces traits qu'apparaît le demandeur d'asile à tous ceux qui ne savent pas s'ils proposent leur 
aide à un réfugié en puissance ou à un futur débouté et qui, dans l'attente, sont les seuls à devoir assumer la contradiction entre deux registres cognitifs entre lesquels finiront par trancher rétrospectivement les autorités : la suspicion et la compassion.

Nous ne voulons en aucun cas signifier que les intervenants sociaux, surtout quand ils sont des professionnels, naviguent quotidiennement entre l'une et l'autre de ces attitudes. Bien au contraire, c'est précisément parce que la plupart d'entre eux ne veulent verser ni dans l'une ni dans l'autre que l'équilibre, et donc la relation d'aide, sont difficiles à tenir : difficile de ne pas s'émouvoir, mais tout aussi risqué de le faire, face à cette femme qui dit avoir été violée mais qui, peut-être, sera déboutée ; difficile de ne pas être désabusé, mais tout aussi dérangeant de l'être lorsqu'on est engagé dans le social, face à ces individus dont on croit savoir qu'ils obtiendront le statut de réfugié " à coup sûr » car ils sont de telle origine et qu' " en ce moment » tous leurs compatriotes l'obtiennent.

On l'aura compris, ce n'est pas en soi la confrontation avec le malheur, ni même avec la dureté d'un monde dans lequel ne règnent pas que la solidarité et la probité, qui rendent la tâche des acteurs de terrain difficile. Nul besoin pour ces derniers d'être amenés à côtoyer les demandeurs d'asile pour avoir une idée de ce que peut être la détresse sociale ou pour faire le deuil d'une vision angélique des relations d'entraide qu'entretiennent ceux qui partagent ce sort. En d'autres termes, ceux qui souffrent ne sont intrinsèquement ni plus ni moins vertueux que tout un chacun et c'est pour cette raison que, comme on aime à le dire dans les écoles de travail social, on ne fait pas du social qu'avec de «bons sentiments ». La proposition peut paraître choquante mais, à bien y réfléchir, elle constitue un garde-fou salutaire contre la tentation de réactiver le clivage qui parcourt toute l'histoire de la gestion de la misère et qui sépare les « bons pauvres », méritants et dociles, des « mauvais pauvres », illégitimes et revendicatifs. Bref, c'est un des défis qu'affronte au quotidien le travail social que d'être en mesure de ne pas céder à cette dichotomie et il suffit de regarder d'un peu près les conditions dans lesquelles travaillent les acteurs de terrain pour mesurer la difficulté de la tâche.

Mais le cadre juridique de l'asile oppose à cette quête permanente de la bonne distance, de la «neutralité bienveillante », une contrainte supplémentaire que l'histoire récente des politiques sociales avait vu disparaître : le principe de la sanction - ici, l'éloignement du territoire - dont la requête n'a pas été jugée recevable. Certes, l'expulsion du demandeur d'asile non reconnu comme réfugié n'est pas la mise au ban que pratiquait le Moyen Âge à l'encontre du vagabond tel que la décrit Castel (1995). À la première n'est pas associée, en tout cas dans les textes qui l'organisent, la réprobation morale qui entourait la seconde. Mais, pour ceux qui accueillent les demandeurs, peu importe qu'il soit dans la logique de la politique de l'asile - et à ce titre légitime - de sélectionner les candidats et dans celle de tout État de réglementer l'entrée et le séjour sur le territoire des étrangers. Le 
résultat est le même pour des travailleurs sociaux qui ne voient pas dans les personnes qu'ils accueillent des justiciables du tribunal administratif mais des usagers au-dessus desquels plane bel et bien le risque de l'éloignement comme sanction apportée à l'illégitimité de leur requête.

On mesure dès lors le trouble de tous ceux qui, parmi les acteurs de terrain, se refusent à être pris, en même temps que les personnes qu'ils accueillent, entre "la potence ou la pitié » ${ }^{1}$, sans pour autant qu'il leur soit toujours possible, ou qu'il leur semble tenable, d'en tirer les motifs d'un engagement militant.

\section{- Conclusion}

Notre enquête met en lumière l'embarras, voire le désarroi dans lequel se trouvent nombre d'acteurs de terrain face à la demande d'asile comme objet d'intervention sociale. On est fondé à y voir, comme le suggèrent les lignes qui précèdent, l'effet d'une contradiction sur le fond entre certains des principes qui prévalent en matière de contrôle des frontières (soumettre la légitimité de la présence des étrangers sur le sol français à des règles sélectives qui, nécessairement, conduisent à certains refus) et ceux qui régissent les pratiques de travail social (œuvrer en faveur de l'«autonomie de la personne $»)$.

Toutefois, si l'incertitude quant à l'issue de la procédure de demande d'asile est fondamentalement déstabilisante pour les travailleurs sociaux qui doivent accompagner des personnes que la loi autorise à vivre légalement sur le territoire pendant cette période d'attente (au nom du droit d'asile) sans pour autant qu'il leur soit permis d'agir dans le sens d'une installation qui reste hypothétique jusqu'à la décision finale (au nom de la maitrise des flux migratoires), il reste que le niveau des moyens financiers et humains engagés par les pouvoirs publics a un rôle essentiel ${ }^{2}$.

Par ailleurs, on peut difficilement isoler les plaintes émises par certains travailleurs sociaux d'un contexte plus global qui n'est pas pour rien dans l'émergence du «stress relationnel » (Weller, 2002) que nous avons cru déceler chez nombre de nos interlocuteurs. Nous pensons ici notamment

1 Pour reprendre le titre du célèbre ouvrage de Geremek (1987).

2 On sait par exemple qu'en CADA les travailleurs sociaux ont le temps de se consacrer à leurs publics, or ils ont dans leurs attributions d'aider à la constitution du dossier de demande d'asile, et que davantage de demandeurs d'asile pourront bénéficier de ces conditions de vie plus favorables (et d'une probabilité d'obtenir le statut de réfugié bien supérieure) si le nombre de places en CPH est suffisant pour accueillir l'ensemble des réfugiés statutaires. Pour prendre un autre exemple, il ne fait guère de doute que la faiblesse des aides financières prévues pour les personnes qui ne sont pas hébergées en CADA est de nature à favoriser, en l'absence de toute possibilité d'occuper un emploi, l'approvisionnement à des sources de revenu illégales. De même, le raccourcissement des délais d'instruction des dossiers auquel se sont attelées les autorités, nécessite des moyens appropriés. 
aux profondes transformations que connaissent les référentiels professionnels et les conditions de travail depuis quelques années et qui semblent aller dans le sens d'un affaiblissement du modèle « artisanal libéral » qui avait permis l'émergence du travail social (Chopart, 2000). Il est à ce titre significatif que la demande d'asile soit appréhendée par beaucoup d'acteurs de terrain comme une cause supplémentaire, parmi d'autres, d'exacerbation de tensions vécues au niveau du front office et que certains n'hésitent pas à relier à un processus de dualisation du système de protection sociale français (Palier, 2002) qui verrait se séparer progressivement la sphère de l'assurance sociale et celle de la solidarité nationale. L'hypothèse d'un « retour de l'assistance », du renforcement d'un « État providence sélectif » privilégiant l'action sur les franges les plus marginalisées de la population dans le cadre de politiques publiques « ciblées » (Rosanvallon, 1995) nourrit en effet depuis une dizaine d'années déjà les interrogations de travailleurs sociaux contraints de s'adapter à « la pression des situations concrètes » (Dourlens, 1998) et qui ont parfois le sentiment de contribuer au développement d'un «droit précaire » (Autès, 1999) imposant aux plus démunis de faire la preuve de leur état de besoin (Fassin, 2000).

Pour le dire plus clairement, s'il est un certain nombre d'éléments qui, comme nous l'avons vu, tendent à constituer le demandeur d'asile en usager atypique, on prendra garde de ne pas surévaluer le caractère inédit des interrogations que ce dernier suscite dans le monde du travail social : il semble bien que ce soit, en filigrane, la logique de l'action humanitaire et, à travers elle, les contraintes que l'assistance comme mode de gestion des populations impose à la relation d'aide, qu'interroge l'émergence de ces nouveaux publics. 


\section{Bibliographie}

AUTÈS M., (1999), « Les territoires politiques de l'action sociale », Informations sociales, $\mathrm{n}^{\circ} 76$.

BORRAZ O., (1999), « Pour une sociologie des dynamiques de l'action publique locale », in Balme R., Faure A., Mabileau A. (dir.), Les nouvelles politiques locales. Dynamiques de l'action publique, Paris, Presses de Sciences-Po.

BOURGEOIS F., EBERMEYER S., SEVIN M., (2004), Demandeurs d'asile, réfugiés statutaires et déboutés dans les dispositifs d'accueil : étude exploratoire dans le département du Rhône, Rapport pour le compte de la DREES du ministère des Affaires sociales.

CASTEL R., (1995), Les métamorphoses de la question sociale, Fayard.

CHOPART J.-N., (2000), « Du travail social à l'intervention sociale »; in Chopart J.-N. (dir.), Les mutations du travail social. Dynamiques d'un champ professionnel, Paris, Dunod.

DARGYTE E., (2002), Vers la mondialisation du vagabondage? Le cas des migrants de l'ex-URSS sur la Côte d'Azur, Mémoire du DEA « Migrations et relations interethniques », université de Nice-Sophia-Antipolis.

DOURLENS C., (1998), Dans les labyrinthes de l'urgence. Le cas de l'Administration sanitaire et sociale, Rapport au ministère du Travail et des Affaires sociales, direction de l'Action sociale, bureau des Études, de la Prospective et de l'Évaluation.

ESPINASSE M.-T., (2002), Pauvreté et précarité des étrangers, Travaux de l'ONPES.

FASSIN D., (2000), « La supplique. Stratégies rhétoriques et constructions identitaires dans les demandes d'aide d'urgence », Annales HSS, septembre-octobre, no 5, p. 953-951.

FRANCE TERRE D'ASILE, (2003), Asile en France, bilan de l'hébergement des demandeurs d'asile et des réfugiés en 2002, Paris, France Terre d'Asile.

FRIGOLI G., JANNOT J., (2004), L'hébergement des demandeurs d'asile comme enjeu local. Éléments d'analyse et perspectives méthodologiques à partir du cas des Alpes-Maritimes, Rapport pour le compte de la DREES du ministère des Affaires sociales.

GEREMEK B., (1987), La potence ou la pitié, Paris, Gallimard.

GUALAMINE F., (2001), « Les faces cachées de la différence culturelle : la construction d'une altérité ambiguë par les travailleurs sociaux », Les Cahiers du CERIEM, n 8, décembre.

IGAS, (2004), Synthèse des bilans de la loi d'orientation du 29 juillet 1998 relative à la lutte contre les exclusions, Paris, IGAS.

MONDOLFO P., (1997), Repenser l'action sociale. Missions, moyens, méthodes, Paris, Dunod.

OFPRA, (2003), Rapport d'activité 2002, Paris, OFPRA.

OFPRA, (2004), Rapport d'activité 2003, Paris, OFPRA.

PALIER B., (2002), Gouverner la sécurité sociale. Les réformes du système français de protection sociale depuis 1945, Paris, PUF.

ROSANVALLON P., (1995), La nouvelle question sociale, Paris, Seuil.

SASSIER M., (1997), « Action sociale et action humanitaire : les avatars d'une liaison dangereuse », in Ridder G. (dir.), Les nouvelles frontières de l'intervention sociale, Paris, L'Harmattan.

WELLER J.-M., (2002), « Stress relationnel et distance au public : de la relation de service à la relation d'aide », Sociologie du travail, $\mathrm{n}^{\circ} 44$. 